\title{
Strong Attractor of Beam Equation with Structural Damping and Nonlinear Damping
}

\author{
Danxia Wang, ${ }^{1}$ Jianwen Zhang, ${ }^{2}$ and Yinzhu Wang ${ }^{3}$ \\ ${ }^{1}$ School of Mathematics, Taiyuan University of Technology, Taiyuan 030024, China \\ ${ }^{2}$ Department of Mathematics, Taiyuan University of Technology, Taiyuan 030024, China \\ ${ }^{3}$ Department of Mathematics, Taiyuan University of Science and Technology, Taiyuan 030024, China
}

Correspondence should be addressed to Jianwen Zhang; jianwen.z2008@163.com.cn

Received 4 December 2012; Revised 18 February 2013; Accepted 27 February 2013

Academic Editor: Shueei M. Lin

Copyright (C) 2013 Danxia Wang et al. This is an open access article distributed under the Creative Commons Attribution License, which permits unrestricted use, distribution, and reproduction in any medium, provided the original work is properly cited.

\begin{abstract}
This paper is mainly concerned with the existence of a global strong attractor for the nonlinear extensible beam equation with structural damping and nonlinear external damping. This kind of problem arises from the model of an extensible vibration beam. By the asymptotic compactness of the related continuous semigroup, we prove the existence of a strong global attractor which is connected with phase space $D\left(\Delta^{2}\right) \times H_{0}^{1}(\Omega) \cap H^{2}(\Omega)$.
\end{abstract}

\section{Introduction}

Global attractor is a basic concept in the study of long-time behavior of nonlinear dissipative evolution equations with various dissipation. There have been many methods to prove the existence of the global attractor. It can be proved by the theory of $\alpha$-contractions of the solution semigroup $S(t)$, such as $[1-3]$ and the reference therein. It can also be proved by the decomposition of the solution semigroup $S(t)$ (see Hale [4], Temam [5], etc.).

In this paper, we use the method of the asymptotically compact property of the solution semigroup $S(t)$ which is different from the method of [1-5] to prove the existence of a strong global attractor for the Kirchhoff type equations with structural damping and nonlinear external damping which arises from the model of the nonlinear vibration beam

$$
\begin{gathered}
u_{t t}+\alpha \Delta^{2} u+\gamma \Delta^{2} u_{t} \\
-\left(\beta+M\left(\int_{\Omega}|\nabla u|^{2} d x\right)+N\left(\int_{\Omega} \nabla u \nabla u_{t} d x\right)\right) \Delta u \\
+g(u)+f\left(u_{t}\right)=h(x), \quad \text { in } \Omega \times R^{+}, \\
u=\Delta u=0 \quad \text { on } \partial \Omega \times R^{+},
\end{gathered}
$$

$$
\begin{gathered}
u(x, 0)=u_{0}(x), \\
u_{t}(x, 0)=u_{1}(x) \quad \text { in } \Omega,
\end{gathered}
$$

where $\alpha, \gamma$, and $\beta$ are all positive constants, $\Omega$ is a bounded domain of $R^{N}$ with smooth boundary $\Gamma=\partial \Omega, M(s), N(s)$, $g(s)$, and $f(s)$ are nonlinear functions specified later, and $h \in L^{2}(\Omega)$ is an external force term. $u(t)$ represents the vertical deflection of the beam, and $u=u(x, t)$ is a real-valued function on $\Omega \times[0,+\infty)$.

In this context of problem (1), based on the vibrating beams equation

$$
u_{t t}+u_{x x x x}-\left(\alpha+\beta \int_{0}^{l}\left|u_{x}(s, t)\right|^{2} d s\right) u_{x x}=0
$$

which is proposed by Woinowsky-krieger [6]; Ma and Narciso [7] considered problem (1) without structural damping and posed a weak global attractor in weak phase space $H_{0}^{2}(\Omega) \times L^{2}(\Omega)$. Eden and Milani [8] considered the existence of exponential attractor for problem (1) with $f(u)=0$ and a linear weak damping $g\left(u_{t}\right)=u_{t}, M(\cdot)$ being a nonlinear function and without structural damping. Ball [9] presented the existence and uniqueness of global solutions for problem (1) with $f=g=h=0, M(\cdot), N(\cdot)$ are all linear functions.

On the other hand, the existence of the attractor for a related problem, with the boundary conditions $u=\Delta u=0$ 
of (2) replaced with $u=\nabla u=0$, was considered by $\mathrm{Ma}$ and Narciso [7], Eden and Milani [8] with a linear damping $u_{t}$ or nonlinear damping $f\left(u_{t}\right)$ without structural damping, respectively. Chueshov and Lasiecka [10] considered a kind of boundary condition which is $u=\Delta u=0$ but without structural damping.

Generally speaking, there have been many works on the long-time behavior for nonlinear beam equations [6-10]. But for the beam equation (1) with structural damping, in strong phase space $D\left(\Delta^{2}\right) \times H_{0}^{1}(\Omega) \cap H^{2}(\Omega)$, the global solutions and the strong global attractor have not still been proved until now.

The outline of this paper is arranged as follows: in Section 2 we give the existence and uniqueness of global solutions in space $C\left(R^{+} ; D\left(\Delta^{2}\right) \times H_{0}^{1}(\Omega) \cap H^{2}(\Omega)\right)$, in Section 3 we give the boundedness of solutions in phase space $D\left(\Delta^{2}\right) \times H_{0}^{1}(\Omega) \cap H^{2}(\Omega)$, and finally in Section 4, we give the proof of the existence of a strong global attractor in phase space $D\left(\Delta^{2}\right) \times H_{0}^{1}(\Omega) \cap H^{2}(\Omega)$.

\section{Some Assumptions and Existence of Global Solution}

In (1), we assume that damping term and the source term are in the form of

$$
f\left(u_{t}\right)=\left|u_{t}\right|^{r} u_{t}, \quad g(u)=|u|^{\rho} u
$$

with

$$
\begin{gathered}
0<\rho, \quad r \leq \frac{2}{N-2} \quad \text { if } N \geq 3, \\
\rho, r>0 \quad \text { if } N=1,2 .
\end{gathered}
$$

We assume that the nonlinear functions $M, N: R^{+} \rightarrow$ $R^{+}$are all class $C^{1}$, and satisfying $M(0)=0, N(0)=0$ and

$$
\begin{gathered}
M(s) s \geq \widehat{M}(s), \quad \text { where } \widehat{M}(s)=\int_{0}^{s} M(z) d z, \\
M(s) \geq 2 s ; \\
N(s) \geq s, \quad \forall s \in R .
\end{gathered}
$$

The functions $f, g: R \rightarrow R$ are also class $C^{1}$, with $f(0)=$ $g(0)=0, \alpha_{1} \leq f^{\prime}(v) \leq \alpha_{2}$, and $\left|g^{\prime}(u)\right| \leq k_{0}\left(1+|u|^{\rho}\right)$ for all $u, v \in R$, where $\alpha_{1}, \alpha_{2}$, and $k_{0}$ are all constants. There also exists constants $k_{5}, k_{6}$ such that

$$
\begin{array}{ll}
|f(u)-f(v)| \leq k_{5}\left(1+|u|^{r}+|v|^{r}\right)|u-v|, & \forall u, v \in R, \\
|g(u)-g(v)| \leq k_{6}\left(1+|u|^{\rho}+|v|^{\rho}\right)|u-v|, & \forall u, v \in R .
\end{array}
$$

In addition, nonlinear function $g(\cdot)$ also satisfies

$$
\begin{gathered}
\varphi(u)+\frac{\alpha-\varepsilon \gamma}{8}\|u\|^{2} \geq-k_{1}, \\
\int_{\Omega} g(u) u d x-C_{1} \varphi(u)+\frac{\alpha}{4}\|u\|^{2} \geq-k_{2},
\end{gathered}
$$

where $\varphi(u)=\int_{\Omega} G(u) d x, G(u)=\int_{\Omega} g(u) d u$, and $k_{1}, k_{2}$ are all constants, $C_{1} \geq 1$.

Our analysis is based on the following Sobolev spaces: $H=L^{2}(\Omega), V=H_{0}^{1}(\Omega) \cap H^{2}(\Omega)$, with the usual inner products and norms as follows, respectively:

$$
\begin{array}{r}
(u, v)=\int_{\Omega} u v d x, \quad|u|=(u, u)^{1 / 2}, \quad \forall u, v \in L^{2}(\Omega), \\
(\Delta u, \Delta v)=\int_{\Omega} \Delta u \Delta v d x, \quad\|u\|=(\Delta u, \Delta u)^{1 / 2}, \\
\forall u, v \in H_{0}^{1}(\Omega) \cap H^{2}(\Omega) .
\end{array}
$$

Consider $D\left(\Delta^{2}\right)=\left\{u \mid u \in V, u \in H^{4}(\Omega), \Delta^{2} u \in\right.$ $\left.H,\left.\Delta u\right|_{\partial \Omega}=0\right\}$ with the inner products $\left(\Delta^{2} u, \Delta^{2} u\right)$ and the norms $\left|\Delta^{2} u\right|^{2}=\left(\Delta^{2} u, \Delta^{2} u\right)$.

Take $E_{0}=H_{0}^{1}(\Omega) \cap H^{2}(\Omega) \times L^{2}(\Omega)$ and $E=D\left(\Delta^{2}\right) \times$ $H^{2}(\Omega) \cap H_{0}^{1}(\Omega)$ with the inner products and norms as follows, respectively:

$$
\begin{gathered}
\left(y_{1}, y_{2}\right)_{E_{0}}=\left(\Delta u_{1}, \Delta u_{2}\right)+\left(v_{1}, v_{2}\right), \quad|y|_{E_{0}}=(y, y)_{E_{0}}^{1 / 2}, \\
\forall y_{i}=\left(u_{i}, v_{i}\right)^{T}, \quad y=(u, v)^{T} \in E_{0}, \quad i=1,2, \\
\left(y_{1}, y_{2}\right)_{E}=\left(\Delta^{2} u_{1}, \Delta^{2} u_{2}\right)+\left(\Delta v_{1}, \Delta v_{2}\right), \quad|y|_{E}=(y, y)_{E}^{1 / 2}, \\
\forall y_{i}=\left(u_{i}, v_{i}\right)^{T}, \quad y=(u, v)^{T} \in E, \quad i=1,2 .
\end{gathered}
$$

Note that assumption (6) implies that $H_{0}^{1}(\Omega) \cap H^{2}(\Omega) \hookrightarrow$ $H_{0}^{1}(\Omega) \hookrightarrow L^{2(p+1)}(\Omega)$, with $p=\rho$ or $p=r$.

Finally, we assume that $\lambda, \sigma$ are the first eigenvalue of $\Delta^{2}$ and $\Delta$, respectively; then we have

$$
\begin{gathered}
\|u\|^{2} \geq \sigma|u|^{2}, \quad \forall u \in V, \\
\left|\Delta^{2} u\right|^{2} \geq \lambda\|v\|^{2}, \quad \forall u \in D\left(\Delta^{2}\right) .
\end{gathered}
$$

In the following, we state the result of the existence and uniqueness of the solutions for systems (1)-(3).

Theorem 1. Assume that $\left(u_{0}, u_{1}\right) \in E, h \in L^{2}(\Omega)$, and the assumptions of these functions $M(\cdot), N(\cdot), f(\cdot)$, and $g(\cdot)$ hold; then problems (1)-(3) have unique solutions $\left(u, u_{t}\right) \in$ $C\left([0, T] ; D\left(\Delta^{2}\right)\right) \times C\left([0, T] ; H_{0}^{1}(\Omega) \cap H^{2}(\Omega)\right)$ depending continuously on initial data in $E$.

By virtue of Galerkin method, we may prove Theorem 1 combined with the priori estimates of Section 3.

According by Theorem 1 , for any $t>0$, we may introduce the mapping

$$
\{S(t), t \geq 0\}:\left\{u_{0}, u_{1}\right\} \longrightarrow\left\{u(t), u_{t}(t)\right\} .
$$

It maps $E$ into itself, and it enjoys the usual semigroup properties as follows:

$$
\begin{gathered}
S(0)=I, \\
S(t+\tau)=S(t) S(\tau), \quad \forall t \geq 0 .
\end{gathered}
$$


And it is obvious that the map $\{S(t), t>0\}$, for all $t \in R$, is continuous in space $E$. In the following, we will introduce the existence of bounded absorbing set and global attractor in space $E$ for map $\{S(t), t \geq 0\}$.

\section{The Existence of Bounded Absorbing Set in Space $E$}

In this section, we will show boundedness of the solutions for systems (1)-(3).

Theorem 2. Assume that these assumptions of Theorem 1 hold then for the dynamic system determined by problems (1)-(3), there exists the boundary absorbing set in space E.

Proof. Taking the inner products of $v=u_{t}+\varepsilon u$ with both sides of (1) and then making summation, we have

$$
\begin{aligned}
\frac{1}{2} \frac{d}{d t} E & (t)-\varepsilon|v|^{2}+\varepsilon(\alpha-\varepsilon \gamma)\|u\|^{2}+\varepsilon \beta|\nabla u|^{2}+\gamma|v|^{2} \\
& +\varepsilon^{2}(u, v)+\varepsilon \widehat{M}(z)+N(\dot{z}) \dot{z}+(g(u), v) \\
& +\left(f\left(u_{t}\right), v\right)=(h, v),
\end{aligned}
$$

where $\widehat{M}(z)=\int_{0}^{z} M(z) d z, z(t)=|\nabla u|^{2}$ and $\varepsilon$ is fixed at arbitrary time, and here the energy function $E(t)$ is defined on $E_{0}$ by

$$
E(t)=|v|^{2}+(\alpha-\varepsilon \gamma)\|u\|^{2}+\beta|\nabla u|^{2}+\widehat{M}(z)+|\nabla u|^{4} .
$$

Considering the assumption $\int_{\Omega} u g(u) d x-C_{1} \varphi(u)+$ $(\alpha / 4)\|u\|^{2} \geq-k_{2}$, we have

$$
\begin{aligned}
(g(u), v)= & \frac{d}{d t} \varphi(u)+\varepsilon(g(u), u) \\
\geq & \frac{d}{d t} \varphi(u)+\varepsilon C_{1} \varphi(u) \\
& -\frac{\varepsilon \alpha}{4}\|u\|^{2}-\varepsilon k_{2} .
\end{aligned}
$$

With $\left|\varepsilon^{2}(u, v)\right| \leq\left(\varepsilon^{2} / \sigma^{2}\right)\|u\|^{2}+\left(\varepsilon^{2} / 4\right)|v|^{2}$, we have

$$
\begin{aligned}
-\varepsilon|v|^{2} & +\varepsilon(\alpha-\varepsilon \gamma)\|u\|^{2}+\varepsilon \beta|\nabla u|^{2}+\gamma|v|^{2}+\varepsilon^{2}(u, v) \\
\geq & \left(\varepsilon \alpha-\varepsilon^{2} \gamma-\frac{\varepsilon^{2}}{\sigma^{2}}\right)\|u\|^{2}+\left(\gamma \lambda^{2}-\varepsilon-\frac{\varepsilon^{2}}{4}\right)|v|^{2} \\
& +\varepsilon \beta|\nabla u|^{2} .
\end{aligned}
$$

With the assumptions $f(0)=0, f \in C^{1}(R, R)$, and $\alpha_{1} \leq$ $f^{\prime}(v) \leq \alpha_{2}$ and by using Mean Value Theorem and Mean Value inequality, we have

$$
\begin{aligned}
\left(f\left(u_{t}\right), v\right) & =\int f^{\prime}(\xi) v^{2} d x-\varepsilon \int f^{\prime}(\xi) u v d x \\
& \geq\left(\alpha_{1}-\frac{\varepsilon \alpha_{2}}{2}\right)|v|^{2}-\frac{\varepsilon \alpha_{2}}{2 \sigma^{2}}\|u\|^{2}
\end{aligned}
$$

where $\xi$ among 0 and $v-\varepsilon u$. Set

$$
\begin{aligned}
\widetilde{E}(t)= & |v|^{2}+(\alpha-\varepsilon \gamma)\|u\|^{2}+\beta|\nabla u|^{2}+\widehat{M}(z) \\
& +|\nabla u|^{4}+2 \varphi(u)+2 k_{1} .
\end{aligned}
$$

Consider

$$
\begin{aligned}
Y(t)= & \left(\varepsilon \alpha-\varepsilon^{2} \gamma-\frac{\varepsilon^{2}}{\sigma^{2}}-\frac{\varepsilon \alpha}{4}-\frac{\varepsilon \alpha_{2}}{2 \sigma^{2}}\right)\|u\|^{2} \\
& +\left(\gamma \lambda^{2}-\varepsilon-\frac{\varepsilon^{2}}{4}+\alpha_{1}-\frac{\gamma \sigma^{2}}{4}-\frac{\varepsilon \alpha_{2}}{2}\right)|v|^{2} \\
& +\varepsilon \beta|\nabla u|^{2}+\varepsilon \widehat{M}(z)+N(\dot{z}) \dot{z}+\varepsilon C_{1} \varphi(u)+\varepsilon k_{1} .
\end{aligned}
$$

So (15) is transformed into

$$
\frac{1}{2} \frac{d}{d t} \widetilde{E}(t)+Y(t) \leq \frac{1}{\gamma \sigma^{2}}|h|^{2}+\varepsilon k_{2}+\varepsilon k_{1} .
$$

Considering the assumptions $M(s) s \geq \widehat{M}(s), M(s) \geq 2 s$, $N(s)>s,\|u\|^{2} \geq \sigma^{2}|u|^{2}$, and $\left|\Delta^{2} u\right|^{2} \geq \lambda^{2}\|u\|^{2}, C_{1} \geq 1$ and letting $0<\varepsilon<\min \left\{\left(\alpha \sigma^{2}+2 \alpha_{2}\right) /\left(2 \gamma \sigma^{2}+4\right),-\left(3+\alpha_{2}\right)+\right.$ $\left.\sqrt{\left(3+\alpha_{2}\right)^{2}+\left(4 \alpha_{1}+3 \gamma \sigma^{2}\right)}\right\}=\varepsilon_{0}$, we have

$$
\frac{2}{\varepsilon} Y(t)-\widetilde{E}(t)>0
$$

Substituting (23) into (22), we have

$$
\frac{1}{2} \frac{d}{d t} \widetilde{E}(t)+\frac{\varepsilon}{2} \widetilde{E}(t) \leq \frac{1}{\gamma \sigma^{2}}|h|^{2}+\varepsilon k_{2}+\varepsilon k_{1} .
$$

On the one hand, applying the Gronwall inequality to (24), we get

$$
\widetilde{E}(t) \leq \widetilde{E}(0) e^{-\varepsilon t}+\frac{2}{\varepsilon}\left(\frac{1}{\gamma \sigma^{2}}|h|^{2}+\varepsilon k_{2}+\varepsilon k_{1}\right), \quad t \geq 0 .
$$

Note that $\|u(0)\|$ and $\left|u_{t}(0)\right|$ are bounded; then there exists a positive constant $R>0$ such that $\widetilde{E}(0) \leq R^{2}$ is bounded; so

$$
\limsup _{t \rightarrow \infty} \widetilde{E}(t) \leq \rho_{0}^{2}=\frac{2}{\varepsilon}\left(\frac{1}{\gamma \sigma^{2}}|h|^{2}+\varepsilon k_{2}+\varepsilon k_{1}\right) .
$$

On the other hand, considering that $\varphi(u)+((\alpha-\varepsilon \gamma) / 8)\|u\|^{2} \geq$ $-k_{1}$, fixing $\mu_{0}>\rho_{0}$, and assuming that $\widetilde{E}(0) \leq R^{2}$, then as $t \geq t_{0}=t_{0}\left(R, \rho_{0}\right)=\left(1 / \varepsilon_{0}\right) \log \left(R /\left(\mu_{0}^{2}-\rho_{0}^{2}\right)\right)$, we have

$$
\widetilde{E}(t) \leq \mu_{0}^{2},
$$

that is,

$$
|v|^{2}+\frac{\alpha-\varepsilon \gamma}{4}\|u\|^{2} \leq \mu_{0}^{2}
$$


Take the inner products by $\Delta^{2} v$ in both sides of (1); then make summation to get

$$
\begin{aligned}
& \frac{1}{2} \frac{d}{d t}\left(\|v\|^{2}+(\alpha-\varepsilon \gamma)\left|\Delta^{2} u\right|^{2}\right)-\varepsilon\|v\|^{2} \\
& \quad-(\beta+M(z(t))+N(\dot{z}(t)))\left(\Delta u, \Delta^{2} v\right) \\
& \quad+\gamma\left|\Delta^{2} v\right|^{2}+\varepsilon^{2}\left(\Delta^{2} u, v\right)+\varepsilon \widehat{M}(z)+N(\dot{z}) \dot{z} \\
& \quad+\left(g(u), \Delta^{2} v\right)+\left(f(v-\varepsilon u), \Delta^{2} v\right)=\left(h, \Delta^{2} v\right) .
\end{aligned}
$$

Considering the continuity of the functions $M^{\prime}(\cdot)$ and $N^{\prime}(\cdot)$, we have

$$
\begin{aligned}
-(\beta & +M(z(t))+N(\dot{z}(t)))\left(\Delta u, \Delta^{2} v\right) \\
& \geq-\left(\beta+C_{2} \mu_{0}^{2}+C_{3} \mu_{0}^{2}\right)|\Delta u|\left|\Delta^{2} v\right| \\
& \geq-\frac{\left(\beta+C_{2} \mu_{0}^{2}+C_{3} \mu_{0}^{2}\right) \mu_{0}^{2}}{\gamma}-\frac{\gamma}{4}\left|\Delta^{2} v\right|^{2},
\end{aligned}
$$

where $C_{2}, C_{3}$ are all positive constants. Also

$$
\begin{aligned}
& \varepsilon^{2}\left(\Delta^{2} u, v\right) \geq-\frac{\varepsilon^{2}}{\sigma^{2}}\left|\Delta^{2} u\right|^{2}-\frac{\varepsilon^{2}}{4}\|v\|^{2}, \\
& \left(h, \Delta^{2} v\right)=\frac{d}{d t}\left(h, \Delta^{2} u\right)+\varepsilon\left(h, \Delta^{2} u\right) .
\end{aligned}
$$

In addition, with $\left|g^{\prime}(u)\right| \leq k_{0}\left(1+|u|^{\rho}\right)$, there exists a constant $k_{3}$ such that $|g(u)|_{L^{\infty}} \leq k_{3},\left|g^{\prime}(u)\right|_{L^{\infty}} \leq k_{3}$; so

$$
\begin{aligned}
& \left(g(u), \Delta^{2} v\right) \\
& =\frac{d}{d t}\left(g(u), \Delta^{2} v\right)-\left(g^{\prime}(u) u_{t}, \Delta^{2} u\right)+\varepsilon\left(g(u), \Delta^{2} u\right) \\
& \geq \frac{d}{d t}\left(g(u), \Delta^{2} u\right)+\varepsilon\left(g(u), \Delta^{2} u\right)-\frac{\varepsilon^{2}}{8}\left|\Delta^{2} u\right|^{2}-\frac{2 k_{3}^{2} \mu_{0}^{2}}{\varepsilon^{2}} .
\end{aligned}
$$

Also by using Schwarz and Mean Value inequalities and Mean Value Theorem, we have

$$
\begin{aligned}
& \left(f(v-\varepsilon u), \Delta^{2} v\right) \\
& \quad \leq \frac{\gamma}{4}\left|\Delta^{2} v\right|^{2}+\frac{1}{\gamma} \int\left(f^{\prime}(\xi)\right)^{2}(v-\varepsilon u) d x \\
& \quad \leq \frac{\gamma}{4}\left|\Delta^{2} v\right|^{2}+\frac{\alpha_{2}^{2}}{\gamma}\left(1+3 \varepsilon^{2}\right) \mu_{0}^{2},
\end{aligned}
$$

where $\xi$ among 0 and $v-\varepsilon u$. Set

$$
\begin{aligned}
Y_{1}(t)= & \left(\varepsilon \alpha-\varepsilon^{2} \gamma-\frac{\varepsilon^{2}}{\sigma^{2}}-\frac{\varepsilon^{2}}{8}\right)\left|\Delta^{2} u\right|^{2} \\
& +\left(\frac{\gamma \lambda^{2}}{2}-\varepsilon-\frac{\varepsilon^{2}}{4}\right)\|v\|^{2}+\varepsilon\left(g(u), \Delta^{2} u\right) \\
& +\varepsilon\left(h, \Delta^{2} u\right),
\end{aligned}
$$

and write $M=\left(\alpha_{2}^{2} / \gamma\right)\left(1+3 \varepsilon^{2}\right) \mu_{0}^{2}+\left(\left(\beta+C_{2} \mu_{0}^{2}+C_{3} \mu_{0}^{2}\right) \mu_{0}^{2}\right) / \gamma+$ $2 k_{3}^{2} \mu_{0}^{2} / \varepsilon^{2}$; then (29) is transformed into

$$
\frac{1}{2} \frac{d}{d t} E_{1}(t)+Y_{1}(t) \leq M .
$$

Here the function

$$
E_{1}(t)=\|v\|^{2}+(\alpha-\varepsilon \gamma)\left|\Delta^{2} u\right|^{2}+2\left(g(u), \Delta^{2} u\right)+2\left(h, \Delta^{2} u\right)
$$

is obtained by the energy function being changed slightly.

Let $0<\varepsilon \leq \min \left\{\varepsilon_{0}, 2 \alpha /\left(\gamma+\left(2 / \sigma^{2}\right)+(1 / 4)\right),-3+\right.$ $\left.\sqrt{9+2 \gamma \lambda^{2}}, \alpha /(\gamma+(1 / 8))\right\}$, we have $Y_{1}(t) \geq(\varepsilon / 2) E_{1}(t)$, and so

$$
\frac{1}{2} \frac{d}{d t} E_{1}(t)+\frac{\varepsilon}{2} E_{1}(t) \leq M, \quad \forall t \geq t_{0}(B) .
$$

Then an application of the Gronwall inequality leads to

$$
E_{1}(t) \leq E_{1}(0) e^{\left[-\varepsilon\left(t-t_{0}\right)\right]}+\frac{2 M}{\varepsilon}, \quad \forall t \geq t_{0}(ß) .
$$

If $B \subset B_{E}(0, \rho)$, there exists a positive constant $R_{1}>0$ such that $E_{1}\left(t_{0}\right) \leq R_{1}^{2}$.

Putting $t_{1}$ satisfing $t_{1}-t_{0}>(1 / \varepsilon) \log R_{1}^{2}$, then as $t \geq t_{1}$, we get

$$
E_{1}(t) \leq R_{1}^{2} e^{-\varepsilon \times(1 / \varepsilon) \log R_{1}^{2}}+\frac{2 M}{\varepsilon}=1+\frac{2 M}{\varepsilon} .
$$

So

$$
\begin{aligned}
& \left(\alpha-\varepsilon \gamma-\frac{\varepsilon}{8}\right)\left|\Delta^{2} u\right|^{2}+\|v\|^{2} \\
& \quad \leq \frac{16}{\varepsilon}|h|^{2}+\frac{16}{\varepsilon} k_{3}^{2}|\Omega|+1+\frac{2 M}{\varepsilon} .
\end{aligned}
$$

The global estimate (40) shows the existence of an absorbing set of $S(t)$.

\section{The Existence of Global Attractor in Space $E$}

The general theory [11] indicates that the continuous semigroup $S(t)$ defined on a Banach space $X$ has a global attractor which is connected when the following conditions are satisfied.

(i) There exists a bounded absorbing set $B \subset X$ such that for any bounded set $B_{0} \subset X$,

$$
\operatorname{dist}\left(S(t) B_{0}, B\right) \longrightarrow 0, \quad \text { as } t \longrightarrow+\infty \text {. }
$$

(ii) $S(t)$ is asymptotically compact; that is, for any bounded sequence $\left\{u_{n}\right\}$ in $X$ and $\left\{t_{n}\right\}$ tending to $\infty$, there exists a subsequence $\left\{n^{\prime}\right\}$ such that $\left\{S\left(t_{n^{\prime}}\right) u_{n^{\prime}}\right\}$ is convergent as $n^{\prime} \rightarrow \infty$.

Theorem 3. Under the assumptions of Theorem 1, the continuous semigroup $S(t)$ has a global attractor which is connected to $E$. 
Proof. Let $u, v$ be two solutions of Problems (1)-(3) in space $C\left(R^{+} ; E\right)$ as shown above corresponding to the initial data $\left(u_{0}, u_{1}\right)$ and $\left(v_{0}, v_{1}\right)$ with $\left|\left(u_{0}, u_{1}\right)\right|_{E}^{2}+\left|v_{0}, v_{1}\right|_{E}^{2} \leq R^{2}$, respectively. Then $w=u-v$ satisfies

$$
\begin{aligned}
w_{t t}+\alpha \Delta^{2} w+\gamma \Delta^{2} w_{t}-\beta \Delta w \\
=\left(M\left(|\nabla u|^{2}\right) \Delta u-M\left(|\nabla v|^{2}\right) \Delta v\right) \\
\quad+\left(N\left(\int \nabla u \nabla u_{t} d x\right) \Delta u-N\left(\int \nabla v \nabla v_{t} d x\right) \Delta v\right) \\
\quad-(g(u)-g(v))-\left(f\left(u_{t}\right)-f\left(v_{t}\right)\right),
\end{aligned}
$$

$$
\left|\left(w, w_{t}\right)\right|_{E_{0}}^{2} \leq C\left(\mu_{0}^{2}\right) .
$$

Taking the inner products in both sides of (42) by $w_{t}, A w$, and $A w_{t}$, respectively, we have

$$
\begin{aligned}
\frac{1}{2} \frac{d}{d t}\left(\left|w_{t}\right|^{2}+\alpha|\Delta w|^{2}+\beta|\nabla w|^{2}\right)+\gamma\left|\Delta w_{t}\right|^{2} \\
=\left(M\left(|\nabla u|^{2}\right) \Delta u-M\left(|\nabla v|^{2}\right) \Delta v, w_{t}\right) \\
\quad+\left(N\left(\int_{\Omega} \nabla u \nabla u_{t} d x\right) \Delta u-N\left(\int_{\Omega} \nabla v \nabla v_{t} d x\right) \Delta v, w_{t}\right) \\
\quad+\left(g(u)-g(v)+f\left(u_{t}\right)-f\left(v_{t}\right), w_{t}\right),
\end{aligned}
$$

$$
\begin{aligned}
\frac{1}{2} \frac{d}{d t}( & \left.\gamma\left|\Delta^{2} w\right|^{2}+2\left(\Delta w, \Delta w_{t}\right)\right)+\alpha\left|\Delta^{2} w\right|^{2} \\
& -\beta\left(\Delta w, \Delta^{2} w\right)+\left|\Delta w_{t}\right|^{2} \\
= & \left(M\left(|\nabla u|^{2}\right) \Delta u-M\left(|\nabla v|^{2}\right) \Delta v, \Delta^{2} w\right) \\
& +\left(N\left(\int_{\Omega} \nabla u \nabla u_{t} d x\right) \Delta u-N\left(\int_{\Omega} \nabla v \nabla v_{t} d x\right) \Delta v, \Delta^{2} w\right) \\
& +\left(g(u)-g(v)+f\left(u_{t}\right)-f\left(v_{t}\right), \Delta^{2} w\right),
\end{aligned}
$$$$
\frac{1}{2} \frac{d}{d t}\left(\left|\Delta w_{t}\right|^{2}+\alpha\left|\Delta^{2} w\right|^{2}\right)+\gamma\left|\Delta^{2} w_{t}\right|^{2}-\beta\left(\Delta^{2} w, \Delta w_{t}\right)
$$$$
=\left(M\left(|\nabla u|^{2}\right) \Delta u-M\left(|\nabla v|^{2}\right) \Delta v, \Delta^{2} w_{t}\right)
$$$$
+\left(N\left(\int_{\Omega} \nabla u \nabla u_{t} d x\right) \Delta u-N\left(\int_{\Omega} \nabla v \nabla v_{t} d x\right) \Delta v, \Delta^{2} w_{t}\right)
$$$$
+\left(g(u)-g(v)+f\left(u_{t}\right)-f\left(v_{t}\right), \Delta^{2} w_{t}\right) .
$$

Equation $(46)+\widetilde{k} \times(45)+\widetilde{\widetilde{k}} \times(44)$ yields

$$
\begin{aligned}
& \frac{1}{2} \frac{d}{d t}\left(\left|\Delta w_{t}\right|^{2}+\alpha\left|\Delta^{2} w\right|^{2}\right. \\
& +\tilde{k} \gamma\left|\Delta^{2} w\right|^{2}+2 \widetilde{k}\left(\Delta^{2} w, w_{t}\right) \\
& \left.+\tilde{\widetilde{k}}\left|w_{t}\right|^{2}+\widetilde{\widetilde{k}} \alpha|\Delta w|^{2}+\tilde{\widetilde{k}} \beta|\nabla w|^{2}\right) \\
& +\gamma\left|\Delta^{2} w_{t}\right|^{2}+\widetilde{k} \alpha\left|\Delta^{2} w\right|^{2}+\widetilde{k}\left|\Delta w_{t}\right|^{2}+\tilde{\tilde{k}} \gamma\left|\Delta w_{t}\right|^{2} \\
& =\left(g(u)-g(v)+f\left(u_{t}\right)-f\left(v_{t}\right), \Delta^{2} w_{t}+\tilde{k} \Delta^{2} w+\tilde{\tilde{k}} w_{t}\right) \\
& +\left(M\left(|\nabla u|^{2}\right) \nabla u-M\left(|\nabla v|^{2}\right) \Delta v\right. \\
& +N\left(\int_{\Omega} \nabla u \nabla u_{t} d x\right) \Delta u \\
& \left.-N\left(\int_{\Omega} \nabla v \nabla v_{t} d x\right) \Delta v, \Delta^{2} w_{t}\right) \\
& +\widetilde{k}\left(M\left(|\nabla u|^{2}\right) \Delta u-M\left(|\nabla v|^{2}\right) \Delta v\right. \\
& +N\left(\int_{\Omega} \nabla u \nabla u_{t} d x\right) \Delta u \\
& \left.-N\left(\int_{\Omega} \nabla v \nabla v_{t} d x\right) \Delta v, \Delta^{2} w\right) \\
& +\tilde{\widetilde{k}}\left(M\left(|\nabla u|^{2}\right) \Delta u-M\left(|\nabla v|^{2}\right) \Delta v\right. \\
& +N\left(\int_{\Omega} \nabla u \nabla u_{t} d x\right) \Delta u \\
& \left.-N\left(\int_{\Omega} \nabla v \nabla v_{t} d x\right) \Delta v, w_{t}\right) \\
& +\left(\beta \Delta w, \Delta^{2} w_{t}\right)+\tilde{k} \beta\left(\Delta w, \Delta^{2} w\right) \text {. }
\end{aligned}
$$

Consider that

$$
\begin{aligned}
\left(M\left(|\nabla u|^{2}\right) \Delta u-M\left(|\nabla v|^{2}\right) \Delta v, \Delta^{2} w_{t}\right) \\
=M^{\prime}\left(\eta_{0}\right)|\nabla u|^{2} \int_{\Omega} \Delta w \Delta^{2} w_{t} d x+M^{\prime}\left(\eta_{1}\right) \\
\quad \times \int_{\Omega} \nabla w(\Delta u+\nabla v) d x \int_{\Omega} \Delta v \Delta^{2} w_{t} d x \\
\leq C_{2} \mu_{0}^{2}|\Delta w|\left|\Delta^{2} w_{t}\right|+2 C_{2} \mu_{0}^{2}|\nabla w|\left|\Delta^{2} w_{t}\right| \\
\leq 2 \frac{\left(C_{2} \mu_{0}^{2}+\left(2 C_{2} \mu_{0}^{2} / \sigma\right)\right)^{2}}{\gamma}|\Delta w|^{2}+\frac{\gamma}{8}\left|\Delta^{2} w_{t}\right|^{2}
\end{aligned}
$$




$$
\begin{aligned}
\widetilde{k}( & \left.M\left(|\nabla u|^{2}\right) \Delta u-M\left(|\nabla v|^{2}\right) \Delta v, \Delta^{2} w\right) \\
= & \tilde{k} M^{\prime}\left(\eta_{0}\right)|\nabla u|^{2} \int_{\Omega} \Delta w \Delta^{2} w d x+\widetilde{k} M^{\prime}\left(\eta_{1}\right) \\
& \times \int_{\Omega} \nabla w(\Delta u+\nabla v) d x \int_{\Omega} \Delta v \Delta^{2} w d x \\
\leq & \tilde{k} C_{2}\left(\mu_{0}^{2}|\Delta w|\left|\Delta^{2} w\right|+\frac{2 \mu_{0}^{2}}{\sigma}|\Delta w|\left|\Delta^{2} w\right|\right) \\
\leq & \frac{\left[C_{2} \widetilde{k} \mu_{0}^{2}(1+(2 / \sigma))\right]^{2}}{\widetilde{k} \alpha}|\Delta w|^{2}+\frac{\widetilde{k} \alpha}{8}\left|\Delta^{2} w\right|^{2} ; \\
\widetilde{\tilde{k}}(M & \left.\left(|\nabla u|^{2}\right) \Delta u-M\left(|\nabla v|^{2}\right) \Delta v, w_{t}\right) \\
= & \widetilde{\tilde{k}} M^{\prime}\left(\eta_{0}\right)|\nabla u|^{2} \int_{\Omega} \Delta w w_{t} d x+\widetilde{\widetilde{k}} M^{\prime}\left(\eta_{1}\right) \\
& \times \int_{\Omega} \nabla w(\Delta u+\nabla v) d x \int_{\Omega} \Delta v w_{t} d x \\
& \leq \widetilde{\tilde{k}} C_{2}\left(\mu_{0}^{2}|\Delta w|\left|w_{t}\right|+\frac{2 \mu_{0}^{2}}{\sigma}|\Delta w|\left|w_{t}\right|\right) \\
& \leq \frac{\left[C_{2} \tilde{\widetilde{k}} \mu_{0}^{2}(1+(2 / \sigma))\right]^{2}}{2}|\Delta w|^{2}+\left|w_{t}\right|^{2},
\end{aligned}
$$

where $\eta_{0}$ is among 0 and $|\nabla u|^{2}, \eta_{1}$ is among $|\nabla u|^{2}$ and $|\nabla v|^{2}$, and

$$
\begin{aligned}
&(\left.N\left(\int_{\Omega} \nabla u \nabla u_{t} d x\right) \Delta u-N\left(\int_{\Omega} \nabla v \nabla v_{t} d x\right) \Delta v, \Delta^{2} w_{t}\right) \\
&= N^{\prime}\left(\xi_{0}\right) \int_{\Omega} \nabla u \nabla u_{t} d x \int_{\Omega} \Delta w \Delta^{2} w_{t} d x+N^{\prime}\left(\xi_{1}\right) \\
& \times\left(\int_{\Omega} \nabla v \nabla w_{t} d x+\int_{\Omega} \nabla w \nabla u_{t} d x\right) \\
& \times \int_{\Omega} \Delta v \Delta^{2} w_{t} d x \\
& \leq C_{3}\left[\mu_{0}^{2}|\Delta w|\left|\Delta^{2} w_{t}\right|+\left(\mu_{0}\left|w_{t}\right|+\mu_{0}|\Delta w|\right) \mu_{0}\left|\Delta^{2} w_{t}\right|\right] \\
& \leq \frac{\left(4 C_{3} \mu_{0}^{2}\right)^{2}}{\gamma}|\Delta w|^{2}+\frac{\gamma}{8}\left|\Delta^{2} w_{t}\right|^{2}+\frac{\left(4 C_{3} \mu_{0}^{2}\right)^{2}}{\gamma}\left|w_{t}\right|^{2}, \\
& \widetilde{k}(\left.N\left(\int_{\Omega} \nabla u \nabla u_{t} d x\right) \Delta u-N\left(\int_{\Omega} \nabla v \nabla v_{t} d x\right) \Delta v, \Delta^{2} w\right) \\
&= \widetilde{k} N^{\prime}\left(\xi_{0}\right) \int_{\Omega} \nabla u \nabla u_{t} d x \int_{\Omega} \Delta w \Delta^{2} w d x+\widetilde{k} N^{\prime}\left(\xi_{1}\right) \\
& \quad \times\left(\int_{\Omega} \nabla v \nabla w_{t} d x+\int_{\Omega} \nabla w \nabla u_{t} d x\right) \\
& \quad \times \int_{\Omega} \Delta v \Delta^{2} w d x \\
& \leq \widetilde{k} C_{3}\left[\mu_{0}^{2}|\Delta w|\left|\Delta^{2} w\right|+\left(\mu_{0}\left|w_{t}\right|+\mu_{0}|\Delta w|\right) \mu_{0}\left|\Delta^{2} w\right|\right] \\
& \leq \frac{\left(4 C_{3} \mu_{0}^{2}\right)^{2} \widetilde{k}}{\alpha}|\Delta w|^{2}+\frac{\tilde{k} \alpha}{8}\left|\Delta^{2} w\right|^{2}+\frac{\left(4 C_{3} \mu_{0}^{2}\right)^{2} \tilde{k}}{\alpha}\left|w_{t}\right|^{2},
\end{aligned}
$$

$$
\begin{aligned}
\tilde{\widetilde{k}}( & \left.N\left(\int_{\Omega} \nabla u \nabla u_{t} d x\right) \Delta u-N\left(\int_{\Omega} \nabla v \nabla v_{t} d x\right) \Delta v, w_{t}\right) \\
= & \widetilde{\widetilde{k}} N^{\prime}\left(\xi_{0}\right) \int_{\Omega} \nabla u \nabla u_{t} d x \int_{\Omega} \Delta w w_{t} d x+\widetilde{\widetilde{k}} N^{\prime}\left(\xi_{1}\right) \\
& \times\left(\int_{\Omega} \nabla v \nabla w_{t} d x+\int_{\Omega} \nabla w \nabla u_{t} d x\right) \\
& \times \int_{\Omega} \Delta v w_{t} d x \\
\leq & \widetilde{\widetilde{k}} C_{3}\left[\mu_{0}^{2}|\Delta w|\left|w_{t}\right|+\left(\mu_{0}\left|w_{t}\right|+\mu_{0}|\Delta w|\right) \mu_{0}\left|w_{t}\right|\right] \\
\leq & \widetilde{\widetilde{k}} C_{3} \mu_{0}^{2}|\Delta w|^{2}+\widetilde{\widetilde{k}} C_{3} \mu_{0}^{2}\left|w_{t}\right|^{2}+\widetilde{\widetilde{k}} C_{3} \mu_{0}^{2}\left|w_{t}\right|^{2},
\end{aligned}
$$

where $\xi_{0}$ is among 0 and $\int_{\Omega} \nabla u \nabla u_{t} d x, \quad \xi_{1}$ is among $\int_{\Omega} \nabla u \nabla u_{t} d x$ and $\int_{\Omega} \nabla v \nabla v_{t} d x$.

Also considering $|g(u)-g(v)| \leq k_{6}\left(1+|u|^{\rho}+|v|^{\rho}\right) \mid u-$ $v \mid$ for all $u, v \in R,|f(u)-f(v)| \leq k_{5}\left(1+|u|^{r}+|v|^{r}\right)|u-v|$, for all $u, v \in R$, and $\rho /(2(\rho+1))+1 /(2(\rho+1))+(1 / 2)=$ $1, r /(2(r+1))+1 /(2(r+1))+(1 / 2)=1$, by Hölder inequality we have

$$
\begin{aligned}
& -\left(g(u)-g(v)+f\left(u_{t}\right)-f\left(v_{t}\right), \Delta^{2} w_{t}\right) \\
& \leq k_{6} \int_{\Omega}\left(1+|u|^{\rho}+|v|^{\rho}\right)|w|\left|\Delta^{2} w_{t}\right| d x \\
& +k_{5} \int_{\Omega}\left(1+\left|u_{t}\right|^{r}+\left|v_{t}\right|^{r}\right)\left|w_{t}\right|\left|\Delta^{2} w_{t}\right| d x \\
& \leq k_{6}\left[\int_{\Omega}\left(1+|u|^{\rho}+|v|^{\rho}\right)^{2(\rho+1) / \rho} d x\right]^{\rho /(2(\rho+1))} \\
& \times|w|_{2(\rho+1)}\left|\Delta^{2} w_{t}\right| \\
& +k_{5}\left[\int_{\Omega}\left(1+\left|u_{t}\right|^{r}+\left|v_{t}\right|^{r}\right)^{2(r+1) / r} d x\right]^{r /(2(r+1))} \\
& \times\left|w_{t}\right|_{2(r+1)}\left|\Delta^{2} w_{t}\right| \\
& \leq C\left(\mu_{0}\right)|\nabla w|\left|\Delta^{2} w_{t}\right|+C\left(\mu_{0}\right)\left|\nabla w_{t}\right|\left|\Delta^{2} w_{t}\right| ; \\
& -\left(g(u)-g(v)+f\left(u_{t}\right)-f\left(v_{t}\right), \widetilde{k} \Delta^{2} w\right) \\
& \leq \widetilde{k} k_{6} \int_{\Omega}\left(1+|u|^{\rho}+|v|^{\rho}\right)|w|\left|\Delta^{2} w\right| d x \\
& +\widetilde{k} k_{5} \int_{\Omega}\left(1+\left|u_{t}\right|^{r}+\left|v_{t}\right|^{r}\right)\left|w_{t}\right|\left|\Delta^{2} w\right| d x \\
& \leq \tilde{k} k_{6}\left[\int_{\Omega}\left(1+|u|^{\rho}+|v|^{\rho}\right)^{2(\rho+1) / \rho} d x\right]^{\rho /(2(\rho+1))} \\
& \times|w|_{2(\rho+1)}\left|\Delta^{2} w\right| \\
& +\widetilde{k} k_{5}\left[\int_{\Omega}\left(1+\left|u_{t}\right|^{r}+\left|v_{t}\right|^{r}\right)^{2(r+1) / r} d x\right]^{r /(2(r+1))} \\
& \times\left|w_{t}\right|_{2(r+1)}\left|\Delta^{2} w\right| \\
& \leq \widetilde{k} C\left(\mu_{0}\right)|w|_{2(\rho+1)}\left|\Delta^{2} w\right|+\widetilde{k} C\left(\mu_{0}\right)\left|w_{t}\right|_{2(r+1)}\left|\Delta^{2} w\right| \\
& \leq \widetilde{k} C\left(\mu_{0}\right)|\nabla w|\left|\Delta^{2} w\right|+\widetilde{k} C\left(\mu_{0}\right)\left|\nabla w_{t}\right|\left|\Delta^{2} w\right| ;
\end{aligned}
$$




$$
\begin{aligned}
& -\left(g(u)-g(v)+f\left(u_{t}\right)-f\left(v_{t}\right), \widetilde{\widetilde{k}} w_{t}\right) \\
& \leq \widetilde{\widetilde{k}} k_{6} \int_{\Omega}\left(1+|u|^{\rho}+|v|^{\rho}\right)|w|\left|w_{t}\right| d x \\
& +\widetilde{\widetilde{k}} k_{5} \int_{\Omega}\left(1+\left|u_{t}\right|^{r}+\left|v_{t}\right|^{r}\right)\left|w_{t}\right|\left|w_{t}\right| d x \\
& \leq \widetilde{\widetilde{k}} k_{6}\left[\int_{\Omega}\left(1+|u|^{\rho}+|v|^{\rho}\right)^{2(\rho+1) / \rho} d x\right]^{\rho /(2(\rho+1))} \\
& \times|w|_{2(\rho+1)}\left|w_{t}\right| \\
& +\widetilde{\widetilde{k}} k_{5}\left[\int_{\Omega}\left(1+\left|u_{t}\right|^{r}+\left|v_{t}\right|^{r}\right)^{2(r+1) / r} d x\right]^{r /(2(r+1))} \\
& \times\left|w_{t}\right|_{2(r+1)}\left|w_{t}\right| \\
& \leq \tilde{\widetilde{k}} C\left(\mu_{0}\right)|w|_{2(\rho+1)}\left|w_{t}\right|+\tilde{\widetilde{k}} C\left(\mu_{0}\right)\left|w_{t}\right|_{2(r+1)}\left|w_{t}\right| \\
& \leq \tilde{\widetilde{k}} C\left(\mu_{0}\right)|\nabla w|\left|w_{t}\right|+\widetilde{\widetilde{k}} C\left(\mu_{0}\right)\left|\nabla w_{t}\right|\left|w_{t}\right| .
\end{aligned}
$$

Setting

$$
\begin{aligned}
E_{2}(t)= & \left|\Delta w_{t}\right|^{2}+\alpha\left|\Delta^{2} w\right|^{2}+\widetilde{k} \gamma\left|\Delta^{2} w\right|^{2}+2 \widetilde{k}\left(\Delta^{2} w, w_{t}\right) \\
& +\widetilde{\tilde{k}}\left|w_{t}\right|^{2}+\tilde{\widetilde{k}} \alpha|\Delta w|^{2}+\widetilde{\widetilde{k}} \beta|\nabla w|^{2}+\beta|\nabla \Delta w|^{2}, \\
Y_{2}(t)= & \frac{\gamma}{2}\left|\Delta^{2} w_{t}\right|^{2}+\frac{\tilde{k} \alpha}{2}\left|\Delta^{2} w\right|^{2}+\frac{\widetilde{k}}{2}\left|\Delta w_{t}\right|^{2} \\
& +\frac{\tilde{\widetilde{k}} \gamma}{2}\left|\Delta w_{t}\right|^{2}+\tilde{k} \beta|\nabla \Delta w|^{2},
\end{aligned}
$$

then substituting (48)-(56) into (47), by Schwarz inequality and Young inequality, and taking $\widetilde{k}>\left(4 C^{2}\left(\mu_{0}\right)\right) / \gamma \sigma^{2}$ and $\tilde{\widetilde{k}} \geq$ $\left(8 \widetilde{k} C^{2}\left(\mu_{0}\right)\right) / \alpha \sigma^{2} \gamma$, we have

$$
\frac{1}{2} \frac{d}{d t} E_{2}(t)+Y_{2}(t) \leq C\left(|\Delta w|^{2}+\left|w_{t}\right|^{2}\right) .
$$

Again setting $\xi=\max \left\{4 / \alpha+4 / \widetilde{k}+4 \gamma / \alpha, 2 / \widetilde{k},\left(4 / \lambda^{2}+\right.\right.$ $\left.\left.4 / \alpha \lambda^{4}\right)(\tilde{\tilde{k}} / \widetilde{k}), 2 / \gamma \lambda^{2}+\left(2 / \gamma \lambda^{2}\right)(\widetilde{k} / \widetilde{\tilde{k}})\right\}$, and considering that $-2 \widetilde{k}\left(\Delta^{2} w, w_{t}\right) \geq-\tilde{k}\left|\Delta^{2} w\right|^{2}-\widetilde{k}\left|w_{t}\right|^{2}$, we have $\xi Y_{2}(t)-E_{2}(t) \geq 0$. On the one hand, from (58) we have

$$
\frac{1}{2} \frac{d E_{2}(t)}{d t}+\frac{1}{\xi} E_{2}(t) \leq C\left(|\Delta w|^{2}+\left|w_{t}\right|^{2}\right) .
$$

Applying the Gronwall inequality to (59), we get

$$
\begin{aligned}
E_{2}(t) \leq & E_{2}(0) e^{-(2 / \xi) t} \\
& +C \int_{0}^{t} e^{-(2 / \xi) t}\left(\|w(\tau)\|^{2}+\left|w_{t}(\tau)\right|^{2}\right) d \tau .
\end{aligned}
$$

On the other hand, with $\left(2 \widetilde{k} w_{t}, \Delta^{2} w\right) \geq-(\widetilde{k} \gamma / 2)\left|\Delta^{2} w\right|^{2}-$ $(4 \tilde{k} / \gamma)\left|w_{t}\right|^{2}$ and setting $\tilde{\widetilde{k}}>4 \widetilde{k} / \gamma$, we get

$$
E_{2}(t) \geq\left|\Delta w_{t}\right|^{2}+\alpha\left|\Delta^{2} w\right|^{2}
$$

Hence

$$
\begin{aligned}
\left|w, w_{t}\right|_{E}^{2} \leq & C E_{2}(0) e^{-2 t / \xi} \\
& +C \int_{0}^{t} e^{-(2 / \xi) t}\left(|\Delta w(\tau)|^{2}+\left|w_{t}(\tau)\right|^{2}\right) d \tau .
\end{aligned}
$$

Now, let $\left\{\left(u_{0 m}, u_{1 m}\right)\right\}$ be a bound sequence in $B_{0} \subset E$, and $\left\{u_{m}(t), u_{m t}(t)\right\}$ the corresponding solutions of problems (1)(3) in $C\left(R^{+}, E\right)$. We assume $t_{n}>t_{m}$. Let $T>0$ and $t_{n}, t_{m}>T$. Then, applying estimate (62) to $w^{m, n}=u_{n}\left(t+t_{n}-T\right)-u_{m}(t+$ $\left.t_{m}-T\right), t \geq 0$, we have

$$
\begin{aligned}
& \mid\left(w^{m, n}, w_{t}^{m, n}\right)_{E}^{2} \\
& \quad \leq C C\left(\mu_{0}\right) e^{-(2 / \xi) t}+C \\
& \quad \times \sup _{0 \leq s \leq t} \mid\left(u_{n}\left(t_{n}-T+s\right)-u_{m}\left(t_{m}-T+s\right)\right), \\
& \left.\quad\left(u_{n t}\left(t_{n}-T+s\right)-u_{m t}\left(t_{m}-T+s\right)\right)\right|_{E_{0}} ^{2} .
\end{aligned}
$$

By taking $t=T$ in the above, we have

$$
\begin{aligned}
& \left|\left(u_{n}\left(t_{n}\right)-u_{m}\left(t_{m}\right), u_{n t}\left(t_{n}\right)-u_{m t}\left(t_{m}\right)\right)\right|_{E}^{2} \\
& \leq C C\left(\mu_{0}\right) e^{-(2 / \xi) T}+C\left(\mu_{0}\right) \\
& \quad \times \sup _{0 \leq s \leq T} \mid\left(u_{n}\left(t_{n}+s\right)-u_{m}\left(t_{m}+s\right)\right), \\
& \left.\quad\left(u_{n t}\left(t_{n}+s\right)-u_{m t}\left(t_{m}+s\right)\right)\right|_{E_{0}} ^{2} .
\end{aligned}
$$

By Sobolev embedding Theorem, for any $T>0$, we can extract a subsequence $\left\{\left(u_{n^{\prime}}, u_{n^{\prime} t}\right\}\right.$ which is convergent in $C\left([0, T] ; E_{0}\right)$ for any $T>0$. For any $\varepsilon>0$, we first fix $T>0$ such that

$$
C C\left(\mu_{0}\right) e^{-(2 / \xi) T}<\frac{\varepsilon}{2},
$$

And, next, taking large $m^{\prime}, n^{\prime}$, we have

$$
\begin{aligned}
C\left(\mu_{0}\right) \sup _{0 \leq s \leq T} \mid\left(u_{n}\left(t_{n}+s\right)-u_{m}\left(t_{m}+s\right),\right. \\
\\
\left.u_{n t}\left(t_{n}+s\right)-u_{m t}\left(t_{m}+s\right)\right)\left.\right|_{E_{0}} ^{2} \leq \frac{\varepsilon}{2} .
\end{aligned}
$$

Then by (62) we have that

$$
\left|\left(u_{n^{\prime}}\left(t_{n^{\prime}}\right)-u_{m^{\prime}}\left(t_{m^{\prime}}\right), u_{n^{\prime} t}\left(t_{n^{\prime}}\right)-u_{m^{\prime} t}\left(t_{m^{\prime}}\right)\right)\right|_{E}^{2} \leq \varepsilon .
$$

We conclude that $S(t)$ is asymptotically compact on $E$. The theorem is now proved.

\section{Acknowledgments}

This work is partially supported by the Natural Science Foundation of China (11172194) and Shanxi Province (2011021002-2 and 2010011008). The authors also wish to give their thanks to the referees for their comments to improve the presentation of this paper. 


\section{References}

[1] A. Eden and V. Kalantarov, "Finite-dimensional attractors for a class of semilinear wave equations," Turkish Journal of Mathematics, vol. 20, no. 3, pp. 425-450, 1996.

[2] A. Eden and V. K. Kalantarov, "On the discrete squeezing property for semilinear wave equations," Turkish Journal of Mathematics, vol. 22, no. 3, pp. 335-341, 1998.

[3] A. Eden, C. Foias, and V. Kalantarov, "A remark on two constructions of exponential attractors for $\alpha$-contractions," Journal of Dynamics and Differential Equations, vol. 10, no. 1, pp. 37-45, 1998.

[4] J. K. Hale, Asymptotic Behavior of Dissipative Systems, vol. 25, American Mathematical Society, Providence, RI, USA, 1988.

[5] R. Temam, Infinite-Dimensional Dynamical Systems in Mechanics and Physics, vol. 68 of Applied Mathematical Sciences, Springer, New York, NY, USA, 1988.

[6] S. Woinowsky-Krieger, Theory of Plates and Shells, McGrawHill, New York, NY, USA, 1959.

[7] T. F. Ma and V. Narciso, "Global attractor for a model of extensible beam with nonlinear damping and source terms," Nonlinear Analysis, vol. 73, no. 10, pp. 3402-3412, 2010.

[8] A. Eden and A. J. Milani, "Exponential attractors for extensible beam equations," Nonlinearity, vol. 6, no. 3, pp. 457-479, 1993.

[9] J. M. Ball, "Stability theory for an extensible beam," Journal of Differential Equations, vol. 14, no. 3, pp. 399-418, 1973.

[10] I. Chueshov and I. Lasiecka, "Long-time behavior of second order evolution equations with nonlinear damping," Memoirs of the American Mathematical Society, vol. 195, no. 912, pp. 12-27, 2008.

[11] Z. J. Yang, "Longtime behavior of the Kirchhoff type equation with strong damping on $R^{N}$," Journal of Differential Equations, vol. 242, no. 2, pp. 269-286, 2007. 


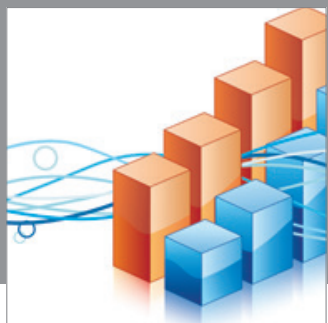

Advances in

Operations Research

mansans

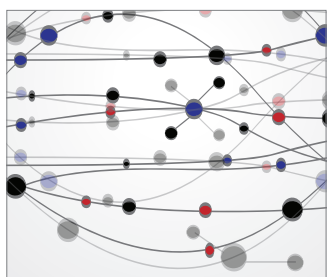

The Scientific World Journal
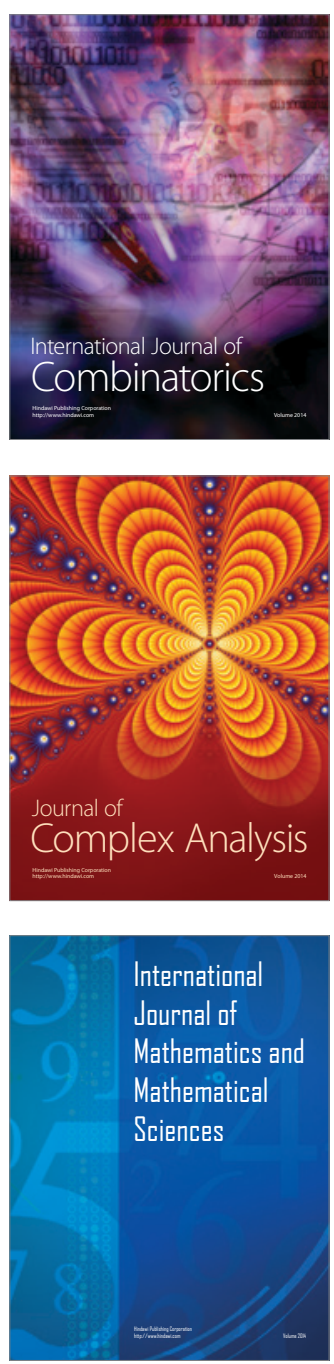
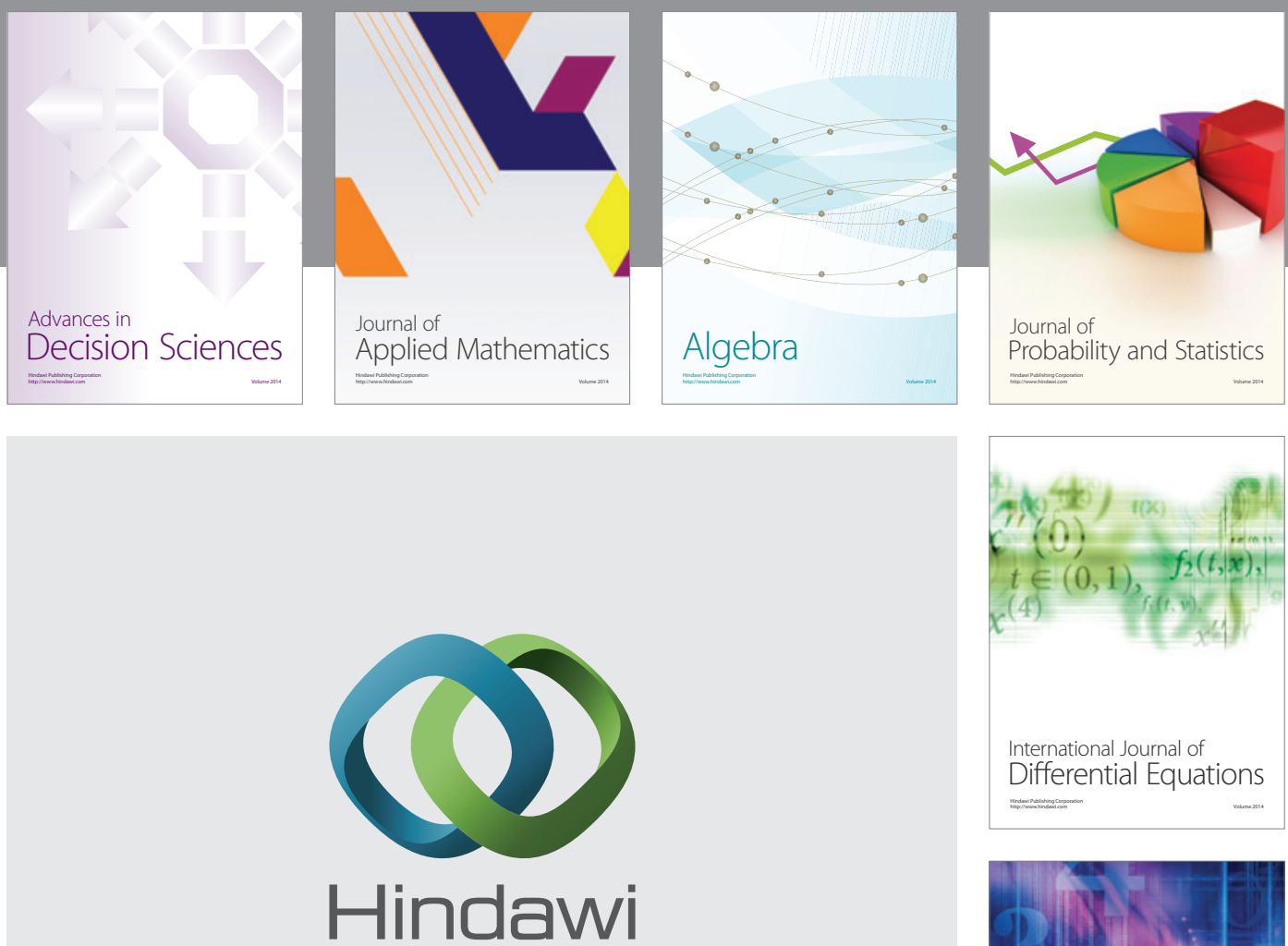

Submit your manuscripts at http://www.hindawi.com
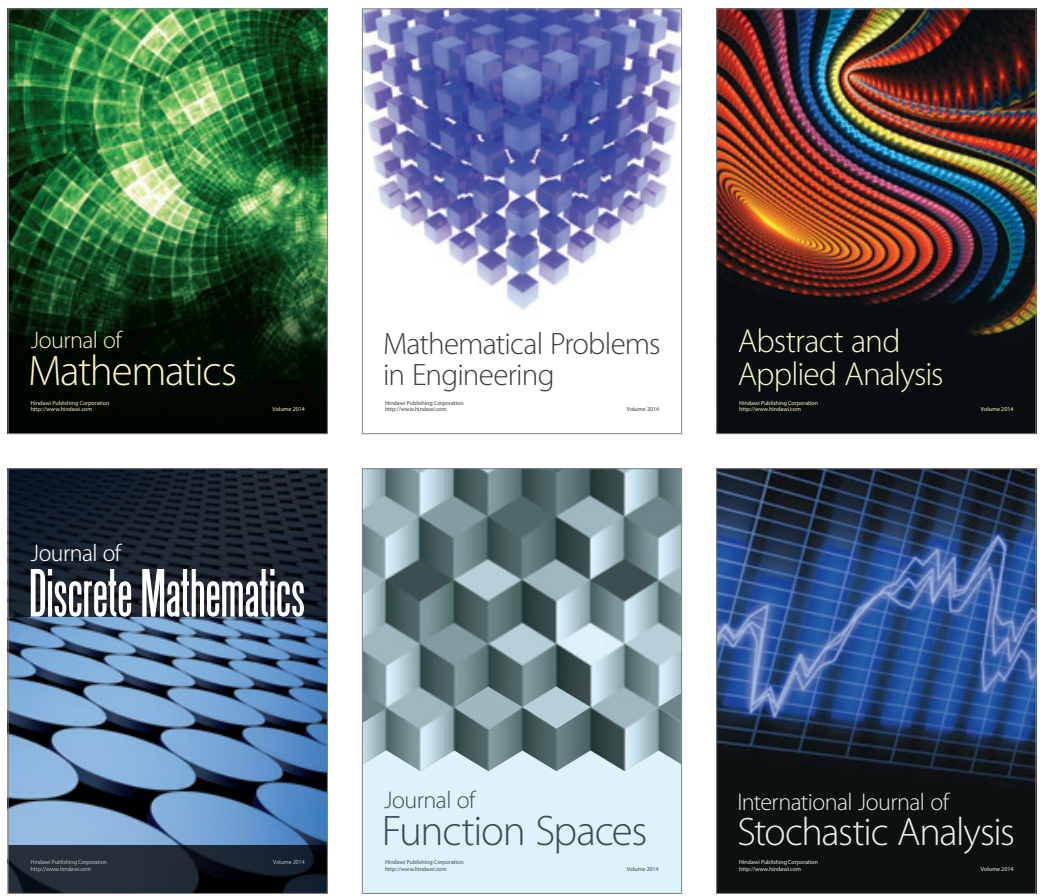

Journal of

Function Spaces

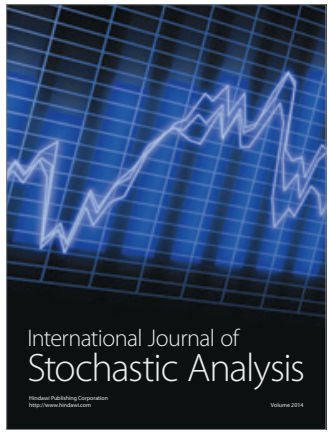

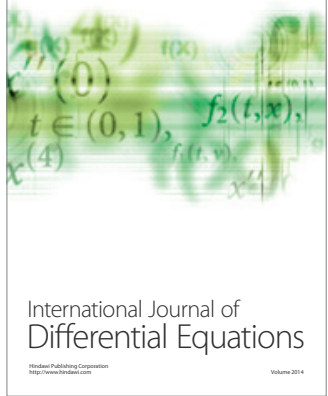
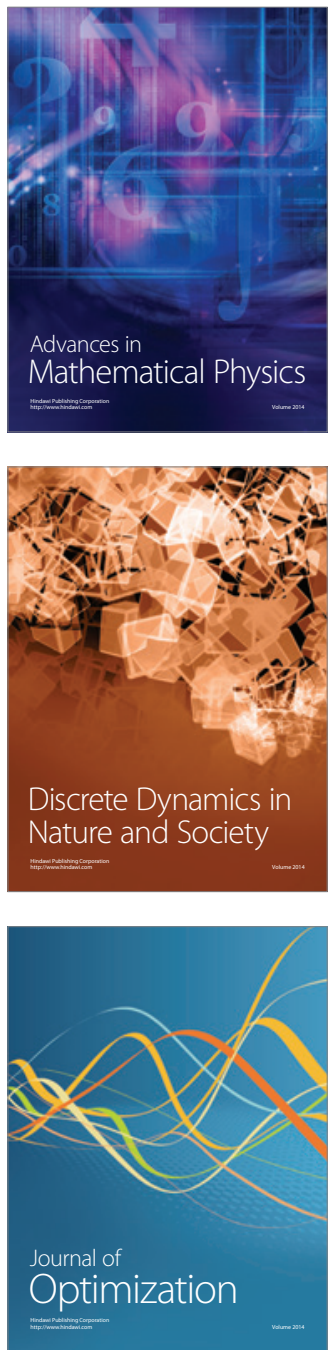